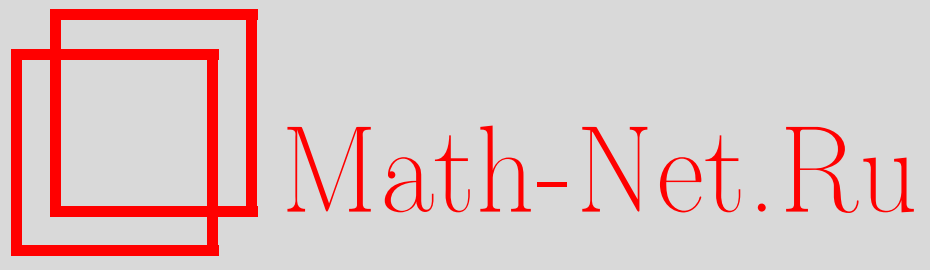

А. Эвнин, Задачи о фокусниках и теоремы Холла и Шпернера, Квант, 2019, номер 2, 14-18

DOI: https://doi.org/10.4213/kvant20190202

Использование Общероссийского математического портала Math-Net.Ru подразумевает, что вы прочитали и согласны с пользовательским соглашением http://www.mathnet.ru/rus/agreement

Параметры загрузки:

IP: 54.237 .206 .68

26 апреля 2023 г., 12:39:26

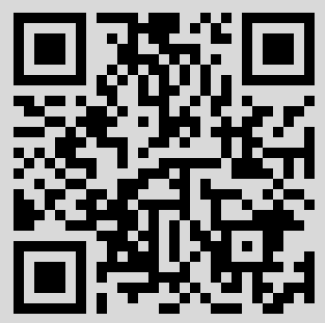




\section{Задачи о фокусниках и теоремы Холла и Шпернера}

\section{А.ЭВНИН}

\section{Теорема Холла}

В популярной математической литературе широкую известность получила следующая задача.

Задача о свадьбах. Имеется множество юношей, каждый из которых знаком с некоторыми девушками. При каких условиях можно одновременно поженить всех юношей так, чтобы каждый из них женился на знакомой ему девушке?

Ответ таков: задача разрешима тогда и только тогда, когда любые $k$ юношей из данного множества знакомы в совокупности не менее чем с $k$ девушками.

Заметим, что необходимость здесь очевидна. Действительно, если для каких-то $k$ юношей число потенциальных невест меньше $k$, то уже этих $k$ юношей одновременно поженить не удастся. Достаточность доказывается не так просто!

Для того чтобы сформулировать задачу о свадьбах на математическом языке, приведем некоторые определения из теории графов [1].

Вершины графа - смежные, если они соединяются ребром. Ребра графа называются смежными, если у них есть общая вершина, и несмежными в противном случае. Попарно несмежные ребра графа образуют паросочетание. Например, в графе на рисунке 1 можно указать несколько паросочетаний из четырех ребер. Одно из них $\left\{a a_{1}, b b_{1}, c c_{1}, d d_{1}\right\}$.

Пусть $G\left(V_{1}, V_{2}\right)$ - двудольный граф с долями $V_{1}$ и $V_{2}$ (в этом графе любое ребро соединяет некоторую вершину из $V_{1}$ с некоторой вершиной из $\left.V_{2}\right)$. Совершенным паросочетанием из $V_{1}$ в $V_{2}$ называется паросочетание в этом графе, покрываю-

DOI: https://doi.org/10.4213/kvant20190202

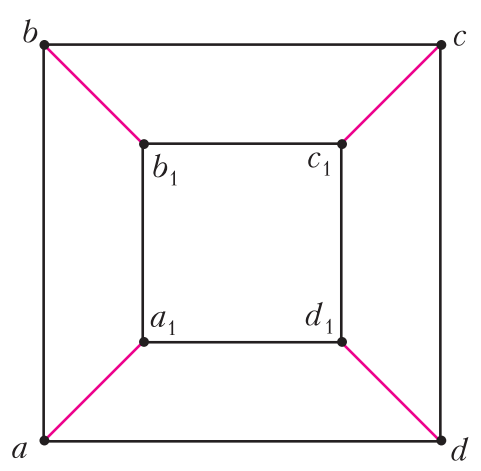

Pnc. 1

щее $V_{1}$ (т.е. для всякой вершины из $V_{1}$ в паросочетании найдется выходящее из нее ребро). Например, на рисунке 2 изображен граф с долями $V_{1}=\left\{x_{1}, x_{2}, x_{3}\right\}$ и

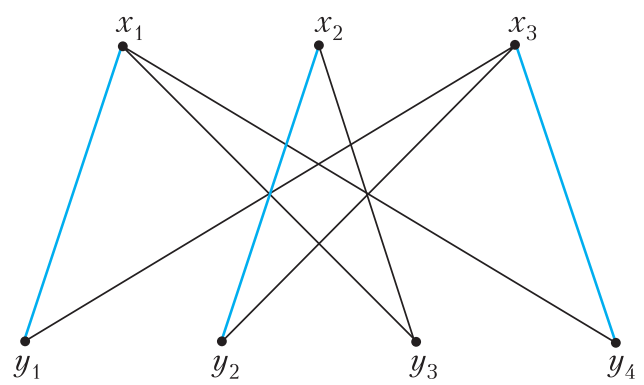

Pис. 2

$V_{2}=\left\{y_{1}, y_{2}, y_{3}, y_{4}\right\}$. Ребра $x_{1} y_{1}, x_{2} y_{2}$ и $x_{3} y_{4}$ образуют совершенное паросочетание из $V_{1}$ в $V_{2}$.

Пусть $G$ - граф с множеством вершин $V$, а $A$ - подмножество $V$. Окружением множества $A$ называют множество

$$
\Gamma(A)=\bigcup_{v \in A} \Gamma(v),
$$

где $\Gamma(v)-$ множество вершин, смежных c $v$. 
Напомним также, что мощность конечного множества - это число его элементов.

Теперь решение задачи о свадьбах может быть сформулировано следующим образом.

Теорема 1 (Ф.Холл, 1935 г.). Совершенное паросочетание из $V_{1}$ в $V_{2}$ в двудольном графе $G\left(V_{1}, V_{2}\right)$ существует тогда и только тогда, когда для любого множества $A \subset V_{1}$ мощность его окружения не меньше мощности $A$, m.e. $|\Gamma(A)| \geq|A|$.

Четыре разных способа доказательства теоремы Холла можно найти в книге [2].

\section{Достаточное условие}

В данной статье речь пойдет об одном (простом, но малоизвестном) достаточном условии существования совершенного паросочетания в двудольном графе.

Теорема 2. Пусть в непустом (т.е. имеющем хотя бы одно ребро) двудольном графе $G\left(V_{1}, V_{2}\right)$ степень любой вериинь из доли $V_{1}$ не меньше степени любой вершины из доли $V_{2}$. Тогда в этом графе существует совершенное паросочетание из $V_{1}$ в $V_{2}$.

Доказательство. Пусть $q$ - число, которое не больше степени любой вершины из $V_{1}$ и не меньше степени любой вершины из $V_{2}$. Возьмем произвольные $k$ вершин первой доли $b_{1}, b_{2}, \ldots, b_{k}$ и смежные с ними вершины $g_{1}, g_{2}, \ldots, g_{l}$ второй доли. Рассмотрим порожденный этими $k+l$ вершинами подграф $G^{\prime}$ исходного графа (в $G^{\prime}$ входят указанные вершины и все ребра между ними, имеющиеся в исходном графе). Ясно, что в графе $G^{\prime}$ степень любой вершины из первой доли не меньше $q$, а из второй не больше $q$. Число ребер двудольного графа равно сумме степеней вершин любой из его долей. Поэтому

$$
k q \leq \sum_{i=1}^{k} \operatorname{deg}\left(b_{i}\right)=\sum_{j=1}^{l} \operatorname{deg}\left(g_{j}\right) \leq l q,
$$

откуда $k \leq l$. Таким образом, выполнены условия теоремы Холла о существовании совершенного паросочетания в двудольном графе.
Граф называется регулярным, если степени всех его вершин равны между собой. Назовем двудольный граф полурегулярным, если в каждой доле степени всех ее вершин равны между собой. Извлечем из теоремы 2 следующие следствия.

Следствие 1. В любом непустом регулярном двудольном графе существует совершенное паросочетание.

Следствие 2. В непустом полурегулярном двудольном графе $G\left(V_{1}, V_{2}\right)$ совершенное паросочетание существует тогда и только тогда, когда в доле $V_{1}$ вершин не больше, чем в $V_{2}$.

Доказательство. Необходимость очевидна.

Достаточность. Пусть в $V_{1}$ и $V_{2}$ степени вершин равны $a$ и $b$ соответственно. Подсчитав двумя способами количество ребер в двудольном графе, получим $a\left|V_{1}\right|=b\left|V_{2}\right|$. Учитывая неравенство $\left|V_{1}\right| \leq\left|V_{2}\right|$, отсюда получаем, что $a \geq b$. Осталось воспользоваться теоремой 2 .

\section{Задачи о фокусниках}

Имеется ряд задач, которые легко решаются с помощью теоремы 2 и следствий из нее. Фабула этих задач такова. Зрители предъявляют ассистенту фокусника некоторый случайным образом выбранный объект (например, несколько игральных карт), после чего ассистент по своему выбору как-то изменяет этот объект. Далее фокусник по новому объекту восстанавливает исходный.

Построим двудольный граф $G\left(V_{1}, V_{2}\right)$ следующим образом. Пусть вершины из $V_{1}$ - это всевозможные конфигурации, которые зрители могут предъявить ассистенту фокусника, а вершины из $V_{2}-$ те конфигурации, которые может получить ассистент. Если из конфигурации $a \in V_{1}$ может быть получена конфигурация $b \in V_{2}$, то в графе должно быть ребро $a b$; других ребер в графе нет.

Если в данном графе существует совершенное паросочетание из $V_{1}$ в $V_{2}$, то фокусник с ассистентом, используя его, смогут осуществить фокус: фокусник по конфигурации $b$ однозначно определит конфигурацию $a$. 
Рассмотрим конкретные задачи.

Для решения некоторых из них нужно знать формулы для числа сочетаний $C_{n}^{k}=\frac{n(n-1) \ldots(n-k+1)}{k !}$ (это количество способов выбрать $k$ элементов из $n$ имеющихся, если порядок выбора не важен) и для числа размещений $A_{n}^{k}=n(n-1) \ldots(n-k+1)$ (это количество способов выбрать $k$ элементов из $n$ имеющихся, если порядок выбора важен).

Задача 1. Имеются 27 карточек с числами от 1 до 27. Двое показывают следующий фокус. Первый получает четыре карточки, выбранные случайным образом. Одну из них он убирает, а три оставшиеся выкладывает в ряд. Второй должен назвать спрятанную карточку. Могут ли участники договориться так, чтобы по выложенным карточкам можно было определить спрятанную?

Ответ: да, могут.

Решение. Рассмотрим двудольный граф, в одной доле которого сочетания из 27 по 4 , в другой размещения из 27 по 3 и ребро соединяет размещение с сочетанием, если все элементы размещения входят в сочетание.

Очевидно, граф полурегулярный (на самом деле, даже регулярный). Поскольку $C_{27}^{4}=A_{27}^{3}=27 \cdot 26 \cdot 25$, к графу можно применить следствие 2 . В рассматриваемом двудольном графе существует совершенное паросочетание. Фокусникам осталось договориться о том, какое совершенное паросочетание им использовать.

Задача 2. Фокусник и его помощник показывают следуюший фокус. Фокусник выкладывает колоду из 36 карт и просит двух зрителей - мальчика и девочку - выбрать по две карты. Видя, кто какие карты выбрал, помощник тоже берет две карты из оставшихся и отдает их зрителям. Зрители перемешивают все 6 карт и отдают их фокуснику. Тот объявляет, кто какие карты брал из колодь. Докажите, ито такой фокус действительно возможен.

Решение. Здесь в первой доле графа упорядоченные пары неупорядоченных пар. Например, вершина $(\{1,2\},\{3,4\})$ со- ответствует случаю, когда мальчик выбрал карты с номерами 1 и 2 (можно считать, что карты пронумерованы числами от 1 до 36), а девочка - карты с номерами 3 и 4. Во второй доле графа неупорядоченные шестерки карт. Ребро проводится в случае, когда шестерка получается из четверки добавлением двух карт. В первой доле построенного двудольного полурегулярного графа $C_{36}^{2} \cdot C_{34}^{2}$ вершин, а во второй $C_{36}^{6}$. Очевидно, $\left|V_{1}\right|<\left|V_{2}\right|$. Выполнены условия следствия 2, гарантирующего существование совершенного паросочетания - фокус возможен.

Задача 3. Зритель пишет на доске слева направо 10 иифр. Помощник фокусника закрывает одну из цифр карточкой. После этого входит фокусник и называет закрытую иифру. Докажите, что такой фокус действительно возможен.

Решение. Пусть $V_{1}$ и $V_{2}$ - множества тех «картинок», которые могут увидеть помощник и фокусник соответственно. Рассмотрим двудольный граф $G\left(V_{1}, V_{2}\right)$, ребра которого описывают возможные переходы от картинок из $V_{1}$ к картинкам из $V_{2}$. Требуется доказать, что в этом графе существует совершенное паросочетание.

Если $u \in V_{1}$, то $\operatorname{deg}(u)=10$, поскольку помощник может закрыть любую из 10 записанных зрителем цифр. Если $v \in V_{2}$, то $\operatorname{deg}(v)=10$, поскольку карточка может закрывать любую из цифр от 0 до 9. Согласно следствию 1 , в $G\left(V_{1}, V_{2}\right)$ существует совершенное паросочетание.

Заметим, что в графе довольно много вершин - по $10^{10}$ в каждой доле. Покажем, что фокус осуществим не только теоретически, но и практически.

Пусть места, где записаны цифры, пронумерованы слева направо: $1,2, \ldots, 9,0$. Помощник подсчитывает сумму $S$ всех записанных зрителем цифр и закрывает карточкой место, номер которого является последней цифрой числа $S$. Фокусник вычисляет сумму девяти открытых цифр и, (по положению карточки) зная последнюю цифру суммы десяти цифр, легко определяет закрытую цифру. 
Задача 4. Зритель пишет на доске слева направо п иифр. Помощник фокусника закрывает одну из чифр красной карточкой, другую синей, еще одну зеленой. После этого входит фокусник и называет закрытые иифры. При каком наименьшем $n$ такой фокус возможен?

Ответ: при $n=12$.

Решение. Пусть $V_{1}$ и $V_{2}$ - множества тех «картинок», которые могут увидеть помощник и фокусник соответственно. Рассмотрим двудольный граф $G\left(V_{1}, V_{2}\right)$, ребра которого описывают возможные переходы от картинок из $V_{1}$ к картинкам из $V_{2}$. Граф полурегулярный, $\left|V_{1}\right|=10^{n}$, $\left|V_{2}\right|=n(n-1)(n-2) \cdot 10^{n-3}$. По следствию 2 , для существования совершенного паросочетания из $V_{1}$ в $V_{2}$ необходимо и достаточно, чтобы в доле $V_{2}$ вершин было не меньше, чем в $V_{1}$. Отсюда $n(n-1)(n-2) \geq$ $\geq 10^{3}$, что равносильно неравенству $n \geq 12$. Таким образом, наименьшее значение $n$ равно 12.

Покажем, как фокус осуществить практически при $n \geq 12$. Места, где записаны первые 12 цифр, пронумеруем слева направо: 1, 2, .., 9, 0, 11, 12 (остальные места, если они есть, не нумеруем!). Пусть $S$ - сумма всех записанных зрителем цифр. Будем действовать за помощника следующим образом. Закроем красной карточкой место, номер которого является последней цифрой числа $S$, после чего мысленно удалим это место, а синей карточкой закроем место с номером, равным цифре, которая спрятана под красной карточкой. Затем зеленой карточкой закроем место, номер которого (вычисленный после «удаления» цифр, находящихся под красной и синей карточками) спрятан под синей карточкой.

Теперь фокусник по положению зеленой карточки поймет, какая цифра под синей, а по положению синей карточки - какая цифра под красной. Все цифры, кроме той, которая под зеленой карточкой, уже известны фокуснику, а положение красной карточки говорит о последней цифре суммы всех цифр, записанных зрителем. Поэтому фокусник сможет определить цифру и под зеленой карточкой.

\section{Задачи для самостоятельного решения}

Приведем подборку задач ${ }^{1}$, при решении которых возможно применение теоремы Холла. Некоторые задачи совсем простые (решаются простой ссылкой, например, на следствие 1; нужно лишь увидеть соответствующий двудольный граф!), некоторые предлагались на школьных и студенческих олимпиадах различного уровня. Задачи 11-15 посвящены доказательству и применениям теоремы Шпернера.

1. Имеется бесконечное множество юношей и бесконечное множество девушек. Для любого натурального числа $k$ верно, что любые $k$ юношей знакомы в совокупности не менее чем с $k$ девушками. Верно ли, что всех юношей удастся одновременно поженить на знакомых им девушках? Другими словами, справедлива ли теорема Холла в случае бесконечного графа?

2. Решите задачу 1 в предположении счетности $^{2}$ множеств юношей и девушек.

3. В некотором районе, состоящем из нескольких деревень, число женихов равно числу невест. В каждой деревне общее число женихов и невест не больше половины общего их числа. Докажите, что можно всех переженить так, чтобы в каждой паре жених и невеста были из разных деревень.

4. Однокруговой волейбольный турнир $2 n$ команд продолжался $2 n-1$ дней. Каждый день проходило $n$ игр, каждая команда в один день проводила ровно одну игру. По окончании турнира организаторы решили наградить тортами некоторые команды: за каждый игровой

1 Задача 3 предлагалась на III этапе Российской олимпиады школьников 1995 года, задача 4 - на Putnam Competition 2012 года, задача 14 - на Московской олимпиаде 2017 года, задача 15 - на Открытой олимпиаде ФМЛ 239 в 2004 году. Остальные задачи взяты из сборников $[3,4]$.

2 Множество называется счетным, если существует взаимно однозначное соответствие между ним и множеством натуральных чисел (другими словами, элементы данного бесконечного множества можно пронумеровать натуральными числами). Счетными являются множества натуральных чисел, неотрицательных целых чисел, целых чисел, рациональных чисел. 
день торт вручается какой-то команде, победившей в этот день. Всегда ли организаторы смогут осуществить свой выбор таким образом, чтобы только одна команда осталась без торта?

5. На танцевальном вечере каждый юноша знаком с $k$ девушками, а каждая девушка знакома с $k$ юношами. Докажите, что можно провести $k$ (медленных) танцев так, чтобы каждый участник вечера станцевал со всеми своими знакомыми (противоположного пола).

6. На шахматной доске пометили 16 из 64 клеток так, что на каждой вертикали и каждой горизонтали оказалось по две помеченные клетки. Докажите, что на помеченных клетках можно расставить 8 черных и 8 белых фигур так, чтобы на каждой вертикали и каждой горизонтали стояло по одной белой и одной черной фигуре.

7. Карточная колода из 36 карт раздается на 9 человек по 4 карты каждому. Докажите, чтобы каждый может выложить по одной карте так, что все эти карты были разного достоинства.

8. Выполняя домашнее задание, каждый студент группы решил по 4 задачи. Известно, что каждая задача была решена четырьмя студентами. Докажите, что можно организовать разбор задач так, чтобы каждый студент рассказал решение ровно одной задачи и чтобы все задачи были разобраны (по одному разу).

9. Пусть $G\left(V_{1}, V_{2}\right)$ - двудольный граф; для любого подмножества $A$ доли $V_{1}$ выполняется неравенство $|\Gamma(A)| \geq|A|-d$. Докажите, что максимальная мощность паросочетания в графе $G$ не меньше $\left|V_{1}\right|-d$.

Для дальнейшего нам понадобятся следующие термины. Пусть имеется некоторый набор различных множеств. Говорят, что они образуют цепь, если для любых двух из них одно является подмножеством другого, и антищепь, если для любых двух из них ни одно не является подмножеством другого. Например, множества $\{1\},\{1,3\}$, $\{1,3,4\}$ образуют цепь, а множества $\{1\}$, $\{2,3\},\{3,4\}$ образуют антицепь. Будем считать, что если у нас всего одно множество, то оно одновременно и цепь, и антицепь. Очевидно, что цепь и антицепь не могут пересекаться более чем по одному множеству. Булеан множества - это множество всех его подмножеств.
10. Докажите, что существует разбиение булеана $n$-элементного множества на цепи, каждая из которых содержит множество мощности $[n / 2]$.

11. Докажите теорему Шпернера: максимальная длина антицепи в булеане $n$-элементного множества равна $C_{n}^{[n / 2]}$.

12. Среди 250 сотрудников международной фирмы в любой паре сотрудников каждый знает язык, который не знает другой сотрудник из этой пары. Какое наименьшее возможное число языков знают (в совокупности) сотрудники фирмы?

13. В деревне живут 250 хоббитов. Каждый хоббит живет в отдельном домике. По вечерам они ходят друг к другу в гости. За один вечер каждый хоббит посещает всех, кого можно застать дома, причем если он идет в гости, то у себя дома в этот вечер уже не появляется. За какое наименьшее число вечеров может случиться так, что каждый житель деревни побывает в гостях у всех остальных?

14. Детектив Ниро Вульф расследует преступление. В деле замешаны 80 человек, среди которых один - преступник, еще один - свидетель преступления (но неизвестно, кто это). Каждый день детектив может пригласить к себе одного или нескольких из этих 80 человек, и если среди приглашенных есть свидетель, но нет преступника, то свидетель сообщит, кто преступник. Может ли детектив заведомо раскрыть дело за 12 дней?

15. Пусть $G$ - граф с множеством вершин $V$. Известно, что для любого множества $A \subset V$ мощность его окружения не меньше мощности $A$. Докажите, что в графе $G$ найдется паросочетание, в котором не меньше $|V| / 3$ ребер.

\section{Литература}

1. М.Л.Краснов. Вся высшая математика: учебник. Т.7/М.Л.Краснов, А.И.Киселев, Г.И.Макаренко, Е.В.Шикин, В.И.Заляпин, А.Ю.Эвнин. - М.: URSS, 2017.

2. А.Ю.Эвнин. Вокруг теоремы Холла. - М.: URSS, 2019.

3. А.Ю.Эвнин. Задачник по дискретной математике. 6-е изд. - М.: URSS, 2016.

4. Всероссийские студенческие турниры математических боев. Тула, 2002-2015 г. Ч. II. Тула: Изд-во ТПГУ им. Л.Н.Толстого, 2017.

Первоначальный вариант статьи опубликован в журнале «Математика в школе» (2019, 스2). 